\title{
Serum and Chyle Lipids during Medium-Chain Triglyceride Feeding in a Child with Chylothorax
}

\author{
I. TAMIR, J. O. HUNTER, AUDREY S. FOSBROOKE, and JUNE K. LLOYD \\ From the Institute of Child Health, University of London, and the Hammersmith Hospital, London
}

Because fatty acids with carbon chain lengths below 12 are absorbed almost wholly into the portal venous system, medium-chain triglycerides (MCT), which contain these shorter chain fatty acids, have been used in the treatment of chylothorax and chylous ascites (Fernandes, Van de Kamer, and Weijers, 1955; Hashim et al., 1964; Frank et al., 1966; Poley et al., 1967). In 3 of the 4 patients reported the volume of chylous fluid diminished; in all patients the concentration of lipid in the fluid decreased and only small quantities of mediumchain fatty acids were detected.

This paper reports studies of both chyle and serum lipids in a child with a traumatic chylothorax during treatment with MCT.

\section{Case Report}

A 7-year-old boy was injured in a road accident and admitted to the Plymouth General Hospital (under the care of Dr. J. N. Montgomery), with a ruptured spleen and fractures of his left 10th and 11 th ribs. Splenectomy was performed. The following day he developed a left pleural effusion which was at first blood-stained and later became chylous. The chyle volume was about 1.5 litres daily, and water and electrolyte losses were replaced by intravenous fluid therapy. Because the volume of chyle showed no signs of diminishing after nine days he was transferred to the Hammersmith Hospital (Dr. J. A. Davis).

On admission his general clinical condition was fair; the results of investigations are summarized in Table I. In an attempt to reduce the flow of chyle he was immediately placed on a low fat diet (2-3 g. fat daily). Intravenous therapy was continued, and calcium, magnesium, and potassium supplements were given. Two days after admission MCT administration was started and the results of this aspect of the treatment will be described separately. The daily volume of chyle lost is shown in the Fig. Large amounts of saline and protein had to be infused in order to maintain plasma levels, and necessitated cannulation of the inferior vena cava. Unfortunately deep vein thrombosis occurred in the right

Received October 10, 1967. ileo-femoral vein, and the superior vena cava was then cannulated via the left axilliary vein. The deep vein thrombosis was treated with intravenous heparin; studies of the serum and chyle lipids had been completed before this treatment became necessary. The continued loss of lymphocytes in the chyle, with resulting low levels in the peripheral blood (120-880/cu.mm.), in a child who had already undergone splenectomy, gave cause for concern, and a negative delayed-sensitivity reaction to skin testing with dinitrochlorobenzene suggested inadequate lymphocyte function. An attempt to replace lymphocytes by the intravenous administration of chyle was abandoned after the injection of only $10 \mathrm{ml}$. was followed by abdominal pain and vomiting.

Twenty-four days after admission (33 days after the injury) chyle was still being lost at the rate of 1-2 litres daily and, though the mean daily loss over a three-day

TABLE I

Results of General Investigations on Admission

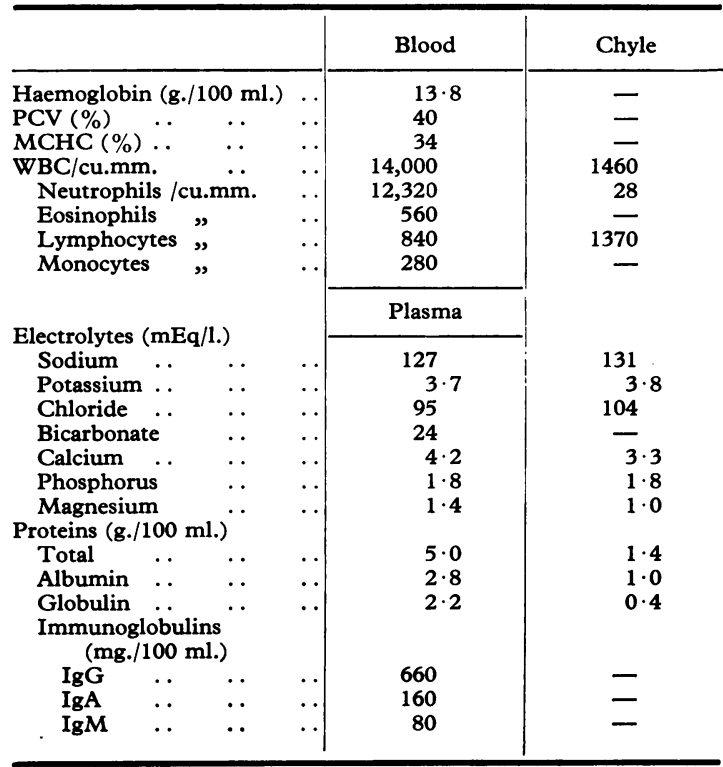




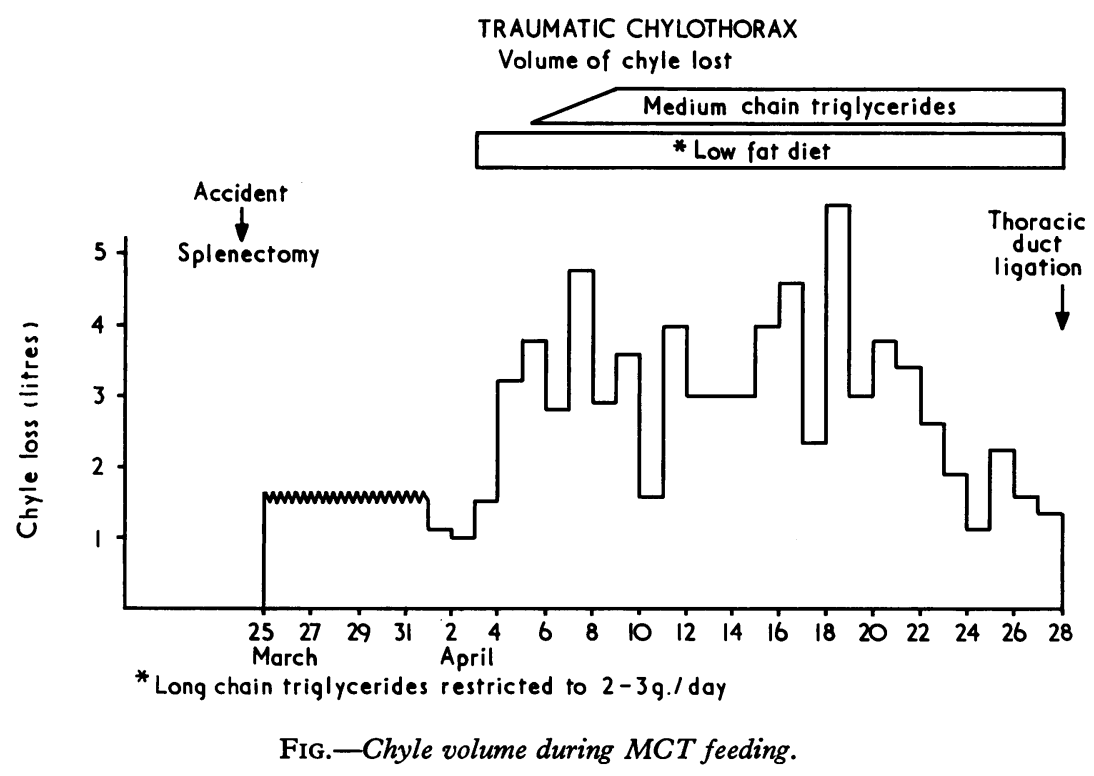

period ( $1 \cdot 6$ litres) was less than formerly $(2 \cdot 6-4 \cdot 0$ litres), it was decided to perform a thoracotomy. At operation (Mr. R. H. Franklin) chyle was seen pouring through a small mediastinal tear in the pleura, and the thoracic duct was ligated above and below the leak at the level of T 7-8. The post-operative progress was uneventful and the patient was discharged from hospital after two weeks.

\section{Studies of Serum and Chyle Lipids}

Venous blood was taken after an overnight fast and a specimen of chyle obtained at the same time from the pleural drain. Lipoprotein electrophoresis was performed by the method of Salt and Wolff (1957); lipids were extracted with ethanol/diethyl ether, $3: 1$; total cholesterol was estimated according to the method of Brown (1961), total phospholipids (lipid phosphorus $\times 25)$ according to the method of Bartlett (1959), and triglyceride according to the method of Freeman (1964). Methyl esters of the plasma non-esterified fatty acids and the triglyceride fatty acids of both chyle and plasma were prepared by the method of I. Tamir and A. S. Fosbrooke (unpublished) and separated by gas-liquid chromatography.

Medium-chain triglycerides were ingested as a $50 \%$ $\mathbf{v} / \mathbf{v}$ emulsion in water, flavoured with fruit juice or tea. The initial quantity given was $12 \mathrm{~g}$. daily and the amount was increased by $12 \mathrm{~g}$. On alternate days to a total daily intake of $48 \mathrm{~g}$. The fatty acid composition of the MCT oil was as follows: caprylic acid (C $8: 0$ ) $75 \%$, capric acid (C $10: 0) 20 \%$, and lauric acid (C 12 : 0) $5 \%$.

\section{Results}

The results of analyses of the serum and chyle lipids and lipoproteins are summarized in Table II. Serum triglyceride concentrations were raised before MCT feeding and fell steadily throughout the period of study. Pre- $\beta$-lipoprotein was detected by electrophoresis when triglyceride levels were high. In the final specimen, which was obtained 7 days after thoracic duct ligation and the resumption of a normal diet, serum triglyceride levels were only slightly above the upper limit of normal and pre- $\beta$ lipoprotein could no longer be detected on paper electrophoresis. Serum cholesterol concentrations were low during MCT feeding and rose on resumption of a normal diet. Concentrations of all lipids in the chyle were low and did not increase significantly on MCT feeding.

The fatty acid composition of the serum triglycerides showed an increased percentage of palmitoleic acid (C $16: 1$ ) and a reduced percentage of linoleic acid (C $18: 2$ ) before MCT feeding. During MCT feeding the amount of palmitoleic acid decreased and levels of linoleic acid remained low. After the resumption of a normal diet the fatty acid distribution was normal.

The major fatty acids of the chyle triglycerides before MCT feeding were palmitic (16:0), palmitoleic $(16: 1)$, and oleic $(18: 1)$. After the 
TABLE II

Lipids and Lipoproteins in Serum and Chyle

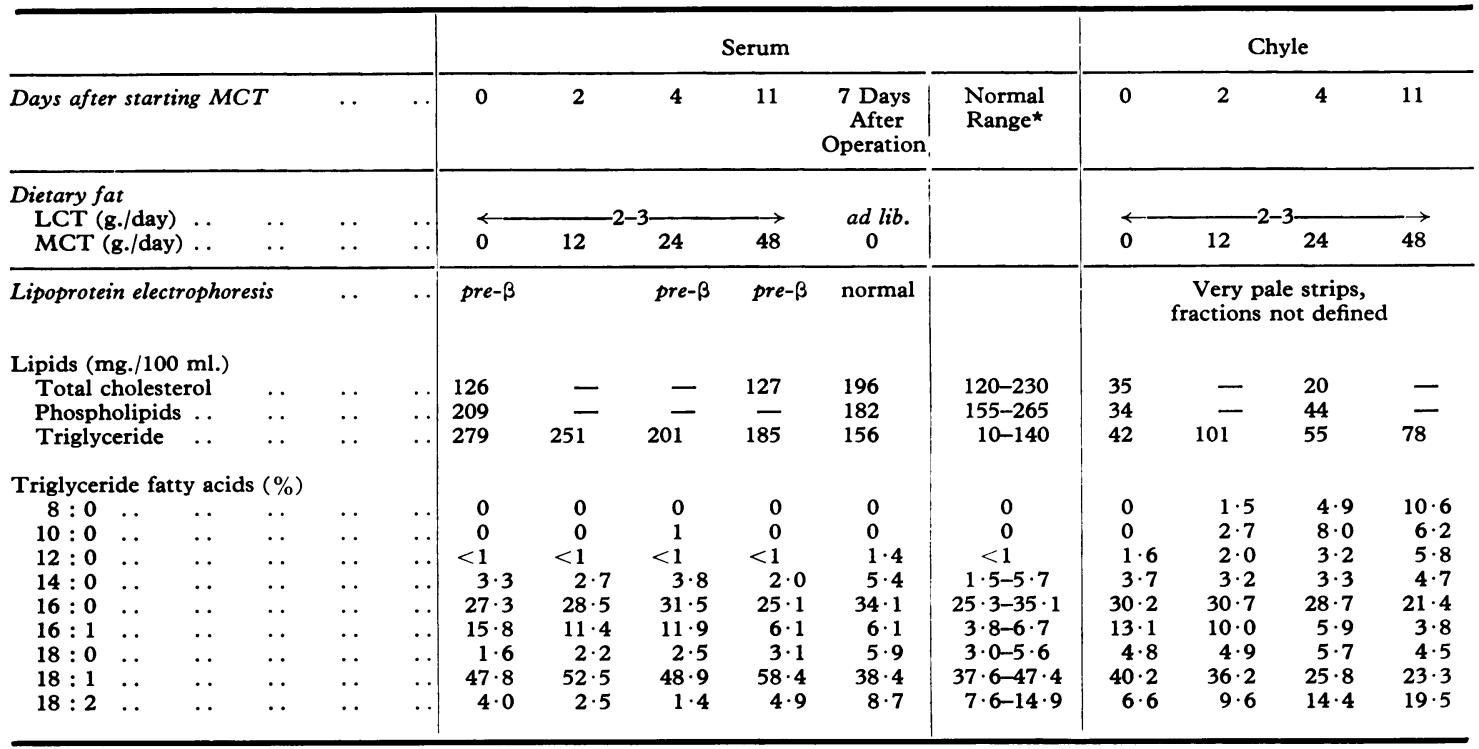

LCT = long-chain triglycerides (containing fatty acids of carbon chain length $>10$ ).

$\star$ Normal values of triglyceride fatty acids obtained from 10 subjects on a normal diet and with normal serum triglyceride concentrations.

introduction of $\mathrm{MCT}$, caprylic $(\mathrm{C} 8: 0)$ and capric (C $10: 0)$ acids appeared in the chyle in increasing concentrations as the dietary intake increased. When expressed as a percentage of the total intake of the individual fatty acids, however, the amount of caprylic acid was less than $1 \%$ of the intake, and that of capric acid between 2 and 3\%. The percentage of palmitic, palmitoleic, and oleic acids decreased during MCT feeding and the percentage of linoleic acid increased.

The serum non-esterified fatty acid composition was similar to that of the serum triglycerides and did not show raised levels of linoleic acid during MCT feeding.

\section{Discussion}

The rate of flow of intestinal lymph is increased during fat absorption (Crandall, Baker, and Graham, 1943), and the feeding of a low fat diet should reduce chyle flow. In our patient, however, the amount of fluid accumulating in the pleural cavity did not lessen when dietary fat was reduced to 2-3 g. daily, though the quantity of triglyceride in the fluid was small (approximately 1-3 g. daily), and its fatty acid composition suggested that it was derived partly from the ingested fat and partly from the serum triglycerides. The feeding of additional fat in the form of MCT in amounts up to $48 \mathrm{~g}$. daily did not increase the volume of fluid; indeed, after 3 weeks of the MCT diet the mean daily loss was reduced to about $50 \%$ of the initial quantity. Furthermore, the concentration of triglyceride in the fluid did not rise significantly, and contained only about $1 \%$ of the ingested MCT (approximately 0.5 g.). The 'failure' of the MCT diet to reduce chyle flow to a sufficient degree to permit spontaneous closure of the tear in the duct cannot, therefore, be attributed to increased fat absorption into the intestinal lymphatics. In the adult the rate of lymph flow in the thoracic duct is about $2 \mathrm{ml} . / \mathrm{kg}$. per $\mathrm{hr}$. (Wetterfors, 1965), and there is no reason to suppose that the flow is substantially different in children (Poley et al., 1967). The accumulation of fluid greatly in excess of this amount in our patient may have been due in part to the effect of continuous drainage with mild suction, and in part to exudate from the pleura itself. The decision to operate after a period of 3 weeks of dietary treatment, despite a slight diminution in the flow of chyle, was taken because of the difficulties in replacing protein loss in a child who had already had a deep vein thrombosis as a consequence of caval catheterization, and because 
of concern over the continuing loss of lymphocytes.

The analysis of the chyle triglycerides confirms that the route of absorption of medium-chain triglycerides is largely, though not entirely, independent of the lymphatic system. Small but significant amounts of both caprylic and capric acids were detected in increasing concentrations as the dietary intake of MCT increased. In this respect our findings are similar to those of Fernandes et al. (1955) and supported by the electronmicroscopical studies of McKay et al. (1967), but at variance with those of Hashim et al. (1964) and Frank et al. (1966) who did not detect any significant amount of medium-chain fatty acids in chyle or ascitic fluid during MCT feeding. Technical problems may contribute to the differences between the findings, the medium-chain triglycerides and fatty acids being water soluble and the methyl esters of the medium-chain fatty acids being highly volatile (I. Tamir and A. S. Fosbrooke, unpublished).

The serum lipid pattern before starting the MCT diet was abnormal. The low level of cholesterol and the reduced percentage of linoleic acid in triglyceride were probably due to the low fat intake and the previous loss of fat in the chyle; the levels of both became normal after thoracic duct ligation and the resumption of normal feeding. The finding of pre- $\beta$-lipoprotein and the fatty acid pattern of the serum triglycerides with an increased percentage of palmitoleic acid suggest that the increased concentration of the serum triglycerides was due to endogenous synthesis from carbohydrate. Similar changes are found in carbohydrate-induced hypertriglyceridaemia (Kaufmann et al., 1966; Levy, Lees, and Fredrickson, 1966), and in our patient the percentage of calories derived from carbohydrate was undoubtedly high, since in addition to his dietary carbohydrate, he had received at least $100 \mathrm{~g}$. glucose daily intravenously. During the period of MCT feeding the steady fall in serum triglyceride concentration, together with a decrease in the percentage of palmitoleic acid, indicated a reduction in the rate of lipogenesis which was probably due to a lower carbohydrate intake.

Our observations that caprylic and capric acids are consistently absent from the serum triglycerides after MCT feeding agree with the findings of Kuo and Huang (1965). These fatty acids are known to be rapidly metabolized by the liver (Schwabe, Bennett, and Bowman, 1964; Valdivieso and Schwabe, 1965).

A fall in linoleic acid concentration in serum triglycerides during MCT feeding is well recognized (Kuo and Huang, 1965), and is presumably due to deficient intake of ordinary dietary fat. The rise in linoleic acid in chyle triglycerides in our patient during MCT feeding is difficult to explain. The composition of the chyle triglycerides before MCT feeding closely resembled that of the serum triglycerides, and one may speculate that as the serum triglyceride levels fell during MCT feeding the contribution of dietary triglycerides to the chyle became more obvious. The total quantity of linoleate in the chyle 11 days after starting MCT was about $600 \mathrm{mg}$., and this amount could have been ingested as part of the low fat diet. We have no evidence that the linoleate was derived by mobilization from adipose tissue, as no increase in linoleic acid was detected in the serum non-esterified fatty acids.

\section{Summary}

A 7-year-old boy with a traumatic chylothorax was treated with a medium-chain triglyceride (MCT) diet. Less than $1 \%$ of the ingested caprylic (C $8: 0$ ) and between 2 and $3 \%$ of the capric acid (C $10: 0)$ appeared in the chyle triglycerides. Neither of these fatty acids was detected in the serum triglycerides. Fasting serum triglyceride concentrations were raised before MCT feeding, and decreased towards normal during MCT feeding. The fatty acid composition of the serum triglycerides suggested that the reduction was due to decreased lipogenesis from carbohydrate. Chyle flow during the period of MCT feeding did not diminish sufficiently to allow spontaneous closure of the tear in the thoracic duct, which was subsequently ligated.

We are grateful to Dr. J. A. Davis and Dr. J. N. Montgomery for permission to study their patient, to Miss D. M. Walker for technical assistance, to Dr. H. P. Sarett of the Mead Johnson Company Ltd. for supplies of MCT oil, and to the Medical Research Council and the Joint Research Board of the Institute of Child Health and The Hospital for Sick Children for financial support.

\section{REFERENCES}

Bartlett, G. R. (1959). Phosphorus assay in column chromatography. f. biol. Chem., 234, 466.

Brown, W. D. (1961). A filtration procedure for determining serum cholesterol with digitonin. Aust.F. exp. Biol. med. Sci., 39, 223.

Crandall, L. A., Baker, S. B., and Graham, D. G. (1943). A study of the lymph flow from a patient with a thoracic duct fistula. Gastroenterology, 1, 1040.

Fernandes, J., Van de Kamer, J. H., and Weijers, H. A. (1955). The absorption of fats studied in a child with chylothorax. $\mathcal{f}$. clin. Invest., 34, 1026.

Frank, B. W., Kern, F., Franks, J. J., and Urban, E. (1966). Failure of medium chain triglycerides in the treatment of persistent chylous ascites secondary to lymphosarcoma. Gastroenterology, $50,677$.

Freeman, N. K. (1964). Simultaneous determination of triglycerides and cholesterol esters in serum by infrared spectrophotometry. F. Lipid Res., 5, 236. 
Hashim, S. A., Roholt, H. B., Babayan, V. K., and Van Itallie, T. B. (1964). Treatment of chyluria and chylothorax with medium chain triglyceride. New Engl. F. Med., 270, 756.

Kaufmann, N. A., Poznanski, R., Blondheim, S. H., and Stein, Y. (1966). Changes in serum lipid levels of hyperlipemic patients following the feeding of starch, sucrose and glucose. Amer. $\mathcal{F}$. clin. Nutr., 18, 261.

Kuo, P. T., and Huang, N. H. (1965). The effect of MCT upon fat absorption and plasma lipid and depot fat of children with cystic fibrosis of the pancreas. F. clin. Invest., 44, 1924.

Levy, R. I., Lees, R. S., and Fredrickson, D. S. (1966). The nature of pre-beta (very low density lipoprotein. ibid., 45, 63 .

McKay, D. G., Kaunitz, H., Csavossy, I., and Johnson, R. E. (1967). Electromicroscope studies of the absorption of lipids. II. Medium chain saturated triglycerides. Metabolism, 16, 127.

Po!ey, J. R., Lesch, P., IHitzig, W. H., and Prader, A. (1967).
Chylöser Asci:es. Untersuchungen über den Eiweisstoffwechsel und über den Einfluss des Nahrumgsfetts auf die Ascites produktion. Helv. paediat. Acta, 22, 81.

Salt, H. B., and Wolff, O. H. (1957). The application of serum lipoprotein electrophoresis in paediatric practice. Arch. Dis. Childh., 32, 404.

Schwabe, A. D., Bennett, L. R., and Bowman, L. P. (1964). Octanoic acid. Absorption and oxidation in humans. f. appl. Physiol., 19, 335.

Valdivieso, V. D., and Schwabe, A. D. (1965). Factors influencing the absorption of a medium chain triglyceride. II. The role of pancreatic juice in the intraluminal phase of absorption. Gastroenterology, 48, 331

Wetterfors, J. (1965). Physiology and Pathophysiology of Plasma Protein Metabolism. Proc. 3rd Symposium. Ed. by H. Koblet. Huber and Bern, Stuttgart. 